\title{
Evaluation of Lightning Current Using Inverse Procedure Algorithm.
}

\begin{abstract}
In this study an inverse procedure algorithm is proposed in the time domain to evaluate lightning return stroke current based on measured electromagnetic fields at an observation point. The proposed method considers on all electromagnetic fields components and can evaluate full shape of current, velocity and constant parameters of current models in contrast with previous studies. In order to validate the proposed method, a set of measured electromagnetic fields are applied on proposed algorithm and the evaluated currents are compared to the corresponding measured current as well as the results are discussed accordingly. The proposed method can be used for preparing of current data bank that is very useful for considering on lightning effects on the power lines and setting appropriate protection levels on the power systems.
\end{abstract}

Keyword: Return stroke current, electromagnetic, lightning 